\title{
Atypical femoral fracture secondary to long-term bisphosphonate use: a case report
}

Sanny Zi Lung Choo, MBBS, Muhammad Hanif Ahmad, MBBS, Shyh Poh Teo, MBChB, FRACP

\begin{abstract}
Bisphosphonates are increasingly used for osteoporosis treatment. Long-term use of bisphosphonates is associated with atypical femoral fractures. We present a case of a 78-year-old woman with this complication and describe the clinical presentation, diagnostic criteria, and management.
\end{abstract}

Key words: Diphosphonates; Femoral fractures; Osteoporosis
Geriatrics and Palliative Unit, Department of Internal Medicine, RIPAS Hospital, Bandar Seri Begawan, Brunei

Correspondence to: Dr Shyh Poh Teo, Geriatrics and Palliative Unit, Department of Internal Medicine, RIPAS Hospital, Bandar Seri Begawan, BA1710, Brunei. Email: shyhpoh.teo@moh.gov.bn

\section{CASE PRESENTATION}

In June 2019, a 78-year-old Filipino woman presented with a 2-week history of left hip pain after a fall from tripping over her walking stick. She had been using a quad-stick to aid walking because of left thigh pain. She walked independently prior to the fall. She had no other history of falls or injuries.

The patient has well-controlled type 2 diabetes mellitus, hypertension, and asthma. 15 years earlier, she had undergone bilateral mastectomy, axillary lymph node clearance, and radiotherapy for breast cancer. She had been taking alendronate, calcium, and vitamin $\mathrm{D}$ for osteoporosis for 15 years.

Radiographs of the patient's left hip and pelvis showed a transverse fracture of the left femoral shaft within cortical thickening in the lateral cortex of the proximal femur (FIGURE a). This was consistent with an atypical femoral fracture associated with bisphosphonate use. Blood test results for calcium, phosphate, electrolyte levels, and renal and hepatic functions were unremarkable.

The femoral fracture was repaired with intramedullary nailing (FIGURE b). The patient was able to bear weight immediately after surgery and she was discharged 5 days later with a walking frame. The patient was advised to discontinue

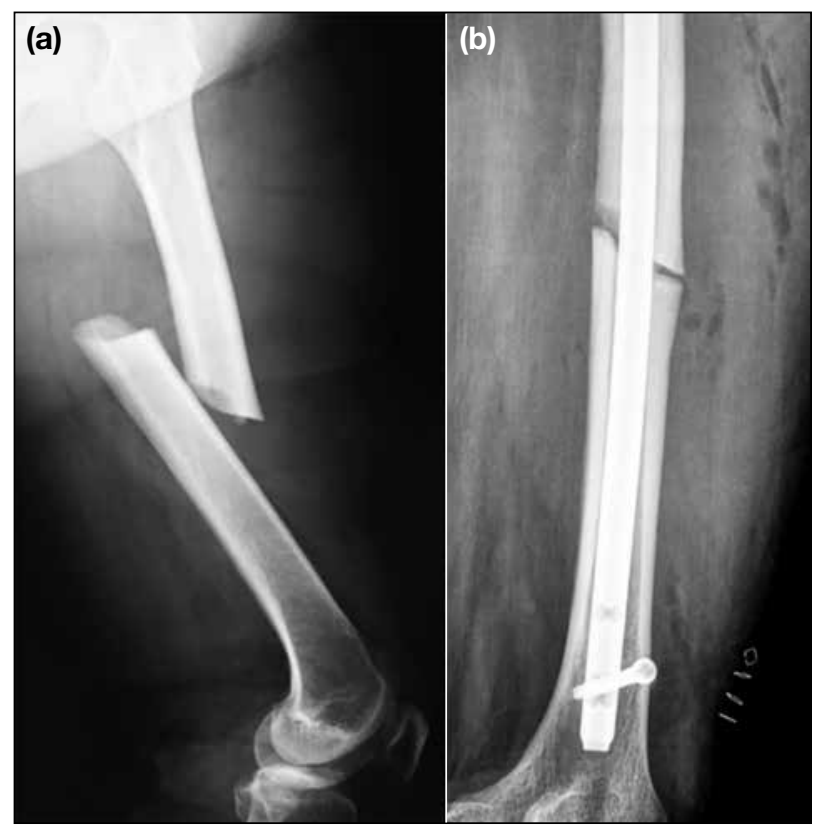

FIGURE. Radiographs of the left femur of a 78-year-old woman showing (a) a non-comminuted fracture along femoral diaphysis with femoral thickening of the medial side, and (b) intramedullary nailing through the fracture site.

bisphosphonates and to use teriparatide to treat her osteoporosis. Radiological evaluation of the contralateral femur was recommended for evidence of atypical fractures. 


\section{DISCUSSION}

Bisphosphonates are commonly prescribed for osteoporosis treatment. However, clinicians should be aware that long-term bisphosphonate use increases the risk of developing atypical femoral fractures. Such fractures are rare, having an estimated incidence of 0.9 to 78 per 100000 person-years. ${ }^{1}$ The estimated incidence increases to 2 per 100000 person-years after 2 years of bisphosphonate use and to 78 per 100000 person-years after 8 years of use. ${ }^{2,3}$

The American Society of Bone and Mineral Research task force diagnostic criteria indicate that atypical femoral fractures are situated along the femoral diaphysis from just distal to the lesser trochanter to just proximal to the supracondylar flare. ${ }^{3}$ In the present case, the major criteria fulfilled were: the fracture occurred with minimal trauma and was non-comminuted; the breakage line originated at the lateral cortex, which was transverse in orientation and oblique as it progresses medially across the femur, extending through both cortices; and the 'beaking' sign, defined as localised periosteal and endosteal thickening of the lateral cortex, was present. The present case also had two minor features: increased cortical thickness of the femoral diaphysis and unilateral prodromal symptoms of dull aching thigh pain.

Several mechanisms have been proposed for the aetiology of atypical fractures, including inadequate repair of stress-induced microfractures, reduced heterogeneity of collagen or mineral content, and increased levels of advanced glycation end-products. ${ }^{4}$

Management of atypical femoral fractures is intramedullary nail fixation and prophylactic surgery for symptomatic incomplete fractures. Calcium and vitamin D should be supplemented. The patient should stop taking bisphosphonates and instead should take bone anabolic agents such as teriparatide to assist healing and restoration of bone quality. ${ }^{5}$
Even after surgical intervention, the prognosis for atypical femoral fractures remains poor, with repeat surgery needed in up to $44 \%$ of cases, with time for consolidation of between 12 and 60 months. $^{6}$

Bisphosphonates should be prescribed for 3 to 5 years, at which time their appropriateness should be reviewed. Bisphosphonates should be stopped in patients evaluated as low or low-to-moderate risk (ie, no incident fractures on therapy, T-score of $\geq-2.0$ and no other major risk factors such as corticosteroid use). If the risk of osteoporotic fractures remains high, bisphosphonates can be continued up to 10 years, with caution. ${ }^{7,8}$

\section{DECLARATION}

The authors have no conflict of interest to disclose.

\section{REFERENCES}

1. Unnanuntana A, Saleh A, Mensah KA, Kleimeyer JP, Lane JM. Atypical femoral fractures: what do we know about them?: AAOS Exhibit Selection. J Bone Joint Surg Am 2013;95:e8 1-13. Crossref

2. Shane E, Burr D, Ebeling PR, et al. Atypical subtrochanteric and diaphyseal femoral fractures: report of a task force of the American Society for Bone and Mineral Research. J Bone Miner Res 2010;25:2267-94. Crossref

3. Shane E, Burr D, Abrahamsen B, et al. Atypical subtrochanteric and diaphyseal femoral fractures: second report of a task force of the American Society for Bone and Mineral Research. J Bone Miner Res 2014;29:1-23. Crossref

4. Flores Santos F, Pinheiro da Silva J, Felicíssimo P. Atypical femoral fractures associated with long-term treatment with bisphosphonates [in Portuguese]. Acta Med Port 2013;26:74650.

5. Adler R, El-Hajj Fuleihan G, Bauer DC, et al. Managing osteoporosis in patients on long-term bisphosphonate treatment: report of a task force of the American Society for Bone and Mineral Research. J Bone Miner Res 2016;31:16-35. Crossref

6. HaYC, Cho MR, Park KH, Kim SY, Koo KH. Is surgery necessary for femoral insufficiency fractures after long-term bisphosphonate therapy? Clin Orthop Relat Res 2010;468:3393-8. Crossref

7. Nieves JW, Cosman F. Atypical subtrochanteric and femoral shaft fractures and possible association with bisphosphonates. Curr Osteoporos Rep 2010;8:34-9. Crossref

8. National Osteoporosis Guideline Group (NOGG) Clinical Guideline for the prevention and treatment of osteoporosis 2017. Available at: https://www.sheffield.ac.uk/NOGG/downloads. html. Accessed 8 September 2019. 